\title{
Sueño y depresión en una muestra de pacientes con dolor crónico
}

\author{
A. Castro ${ }^{1,2,3}$, M. Gili1 ${ }^{1,2,3}$, J.L. Aguilar ${ }^{1,2,4}$, R. Pélaez ${ }^{1,2,4}$ y M. Roca ${ }^{1,2,3}$ \\ ${ }^{1}$ Institut Universitari d'Investigació en Ciències de la Salut (IUNICS). Universidad de les Illes Balears. \\ Palma de Mallorca. ${ }^{2}$ Instituto de Investigación Sanitaria de Palma (IDISPA). Palma de Mallorca. \\ ${ }^{3}$ Red de Actividades Preventivas y Promoción de la Salud en Atención Primaria (RediAPP). \\ ${ }^{4}$ Servicio de Anestesiología, Reanimación y Unidad del Dolor. Hospital Son Llàtzer. Palma de Mallorca
}

Castro A, Gili M, Aguilar JL, Pélaez R, Roca M. Sueño y depresión en una muestra de pacientes con dolor crónico. Rev Soc Esp Dolor 2014; 21(6): 299-306.

\begin{abstract}
Introduction: Sleep disturbances and affective disorders (major depressive disorder and generalized anxiety disorder) are two comorbidities very frequents in patients with chronic pain.

Objective: The main objective is to evaluate sleep quality and the prevalence of major depressive disorder and generalized anxiety disorder in patients with musculoskeletal chronic pain. As secondary objectives: To compare sleep quality and pain severity with different variables and to know the sleep quality predictors.

Material and methods: Transversal descriptive study was made in the Pain Unit from Son Llàtzer Hospital (Palma de Mallorca, Spain). A total of 39 patients were included, all of them older than 18 years old and with musculoskeletal chronic pain. Inclusion was during March, April and May 2013. Excluded were patients younger than 18 years old, those who refuse to participate in the study or/and those who diagnosed with severe psychiatric disorder (dementia, psychosis, alcohol/drug abuse). Pain severity was assessed by a visual analogue scale, major depressive disorder and generalized anxiety disorder was assessed by the International Neuropsychiatric Interview, sleep quality was assessed by the Pittsburgh Questionnaire and depressive symptomatology was assessed by the Montgomery-Asberg Questionnaire.

Results: Chronic pain patients related poor sleep quality. $69.2 \%$ of patients had major depressive disorder and/or $65.5 \%$ had a generalized anxiety disorder. There are not sta-
\end{abstract}

Recibido: 10-09-13.

Aceptado: 15-12-13. tistical differences in quality of sleep between pharmacological groups (potens opioids vs. minor/antiinflamatories). There are statistical differences in some Pittsburgh Questionnaire items: subjective sleep quality, regular efficiency, dreams perturbances, daily dysfunction and total score, as well as, in pain severity: patients with depression had higher scores than patients without depression. The significant predictors of sleep quality were age and depression severity.

Conclusions: These results are in accordance with other studies that show that chronic pain patients suffer poor sleep quality, depression and anxiety. Future researchers are necessary to evaluate these variables, to know the importance of these factors that play a role in this medical condition and to improve clinical management and quality of life of these patients.

Key words: Sleep quality. Depression. Anxiety. Chronic pain.

\section{RESUMEN}

Introducción: los trastornos del sueño y los trastornos afectivos (trastorno depresivo mayor y trastorno de ansiedad generalizada) son dos de las condiciones comórbidas más frecuentes en los pacientes con dolor crónico.

Objetivo: el objetivo principal de este estudio es evaluar la calidad del sueño y la prevalencia del trastorno depresivo mayor y trastorno de ansiedad generalizada en pacientes con dolor crónico osteomuscular. Como objetivos secundarios se señalan: comparar la calidad del sueño y la evaluación del dolor en distintas variables y conocer los predictores de la calidad del sueño.

Material y métodos: se diseñó un estudio descriptivo transversal realizado en la Unidad del Dolor del Servicio de Anestesiología y Reanimación del Hospital Son Llàtzer (Palma de Mallorca). Un total de 39 pacientes participaron en el estudio, todos ellos mayores de edad y con diagnóstico de dolor crónico tipo osteomuscular. La inclusión se realizó durante los meses de marzo, abril y mayo de 2013. Se excluyeron aquellos pacientes menores de 18 años, rechazaban participar en el estudio y/o presentaban un trastorno psiquiátrico grave (demencia, 
psicosis, abuso de alcohol/drogas). Se utilizó un cuestionario de elaboración propia para recoger variables sociodemográficas, de estilo de vida y clínicas, una escala visual analógica para evaluar la intensidad del dolor, la entrevista Neuropsiquiátrica Internacional para determinar diagnóstico de trastorno depresivo mayor y trastorno de ansiedad generalizada, el Cuestionario de Pittsburgh para evaluar la calidad del sueño y la Escala de Depresión Montgomery-Asberg para evaluar la gravedad de la sintomatología depresiva.

Resultados: la muestra refirió una mala calidad del sueño. El $69,2 \%$ de la muestra presentaba un trastorno depresivo mayor y/o un 65,5 \% un trastorno de ansiedad generalizada. No se encontraron diferencias estadísticamente significativas en la calidad del sueño entre los grupos tratados con opioides potentes y tratados con opioides menores/antiinflamatorios. Existen diferencias estadísticamente significativas en determinados ítems del Cuestionario de Pittsburgh: calidad subjetiva del sueño, eficiencia habitual, perturbaciones del sueño, disfunción durante el día y en la puntuación total del cuestionario, así como en la evaluación del dolor en el que los pacientes con depresión presentaban puntuaciones más elevadas respecto a los que no tenían depresión. Los predictores significantes de la calidad del sueño fueron la edad y la gravedad de la depresión.

Conclusiones: estos resultados van en la línea de aquellas investigaciones que muestran que los pacientes con dolor crónico presentan pobre calidad de sueño, depresión y ansiedad. Se necesitan futuras investigaciones para evaluar estas variables, conocer la importancia de los factores implicados en esta condición médica y mejorar el manejo clínico y la calidad de vida de estos pacientes.

Palabras clave: calidad del sueño, trastorno depresivo mayor, trastorno de ansiedad generalizada, dolor crónico.

\section{INTRODUCCIÓN}

El dolor es el síntoma más común de una enfermedad o lesión que obliga a los pacientes a buscar asesoramiento clínico y terapéutico (1). El 29 \% de la población española no hospitalizada sufre algún tipo de dolor $(2,3)$. La Asociación Internacional del Estudio del Dolor (4) lo define como una experiencia sensorial o emocional desagradable, asociada a un daño tisular o potencial, o bien descrita en términos de tal daño. Esta definición de dolor no sólo atiende a los factores fisiológicos, sino que también tiene en cuenta los factores psicológicos y sociales y hace que el enfoque terapéutico sea más ambicioso $(5,6)$. El dolor crónico se define como aquel dolor que persiste más allá del tiempo normal de curación de los tejidos y propone 3 meses como un punto de corte conveniente (4) y en el que se suele agravar por factores ambientales estresantes o psicopatológicos, provocando, en muchas ocasiones, una incapacidad funcional de la persona. (7). El dolor crónico afecta al 20-35\% de la población mundial (8).

Son muchos los trabajos que han relacionado el dolor crónico con otros trastornos, como por ejemplo, con los trastornos del sueño $(9,10)$. Los trastornos de sueño son una de las condiciones comórbidas más frecuentes en pacientes con dolor crónico $(8,11-14)$. Entre un $50 \%$ y un $88 \%$ de pacientes con dolor crónico presenta importantes quejas sobre su calidad de sueño $(15,16)$. Se sugiere que los trastornos del sueño en pacientes con dolor crónico se caracterizan por una interrelación circular, en el que el dolor lleva a trastornos del sueño y estos incrementan la percepción del dolor $(8,12)$. Algunas explicaciones a este fenómeno van en dirección al uso de opioides como posible perturbador del sueño, ya que varios estudios han demostrado que el uso de opioides para manejar el dolor perturba la arquitectura del sueño e inhibe la fase $\operatorname{REM}(8,17,18)$.

Los trastornos afectivos también se han relacionado con el dolor crónico $(12,13,19)$, de hecho, entre un $60 \%$ y un $80 \%$ de personas con dolor crónico también presentan un trastorno afectivo (20). La depresión y la ansiedad son mucho más comunes en pacientes con dolor crónico que en la población general (21), encontrándose síntomas depresivos desde un 22 hasta el $78 \%$ en estos pacientes $(22,23)$. En la relación dolor-depresión, no debe ser obviado el hecho de que si bien el dolor influye en los niveles de depresión, también la depresión determina los niveles de dolor, actuando recíprocamente, de forma parcial o grave, premórbida y/o intermórbida $(20,24)$.

El objetivo principal de este estudio es evaluar la calidad del sueño y la prevalencia del trastorno depresivo mayor y trastorno de ansiedad generalizada en una muestra de pacientes con dolor crónico del tipo osteomuscular. Los objetivos secundarios son: comparar la calidad del sueño entre los pacientes que presentan trastorno depresivo mayor y los que no; comparar la gravedad del dolor entre diferentes grupos de pacientes (trastorno depresivo mayor, trastorno de ansiedad generalizada, comorbilidad, trastorno depresivo mayor y trastorno de ansiedad generalizada); comparar la calidad del sueño entre el grupo de pacientes que toma opioides potentes y el grupo que toma opioides menores o antiinflamatorios para manejar el dolor; y conocer los predictores significativos de la calidad del sueño.

\section{METODOLOGÍA}

\section{Diseño del estudio, muestra y reclutamiento}

Se diseñó un estudio descriptivo transversal realizado en la Unidad del Dolor del Servicio de Anestesiología y Reanimación del Hospital Son Llàtzer (Palma de Mallorca). Un total de 39 pacientes se incluyeron en el estudio. Se seleccionaron aquellos participantes que acudían a la consulta de la Clínica del Dolor y que cumplían los criterios de inclusión previamente establecidos: a) ser mayor de 18 años y b) presentar diagnóstico de dolor crónico tipo osteomuscular. La inclusión se llevó a cabo durante los meses de marzo, abril y mayo de 2013. Se excluyeron los pacientes que: a) eran 
menores de 18 años; b) rechazaban participar en el estudio, y c) presentaban un diagnóstico de trastorno psiquiátrico grave (demencia, psicosis, abuso de alcohol/drogas).

Una vez que los pacientes acudían a la Unidad del Dolor, el médico especialista comprobaba si cumplían los criterios de inclusión. En este caso, se les presentaba el estudio y era el paciente quien decidía sobre su participación. A continuación, uno de los tres psicólogos encargados de las evaluaciones realizaba la entrevista a los participantes incluidos. Las evaluaciones tenían una duración de 30-45 minutos aproximadamente. Todos los participantes firmaron el consentimiento informado.

\section{Instrumentos}

Variables sociodemográficas, de estilo de vida y médicas

Se diseñó un cuestionario de elaboración propia para recoger variables de sexo, edad, lugar de residencia, estado civil, convivencia, nivel de estudios, situación laboral actual, consumo de bebidas alcohólicas y cafeína al día, consumo de tabaco diario, el nivel de actividad física habitual y tratamiento farmacológico.

Valoración del dolor: escala visual analógica (EVA) de la intensidad del dolor

Consiste en una escala numerada del 0 al 10 con dos extremos de intensidad $(0=$ no dolor, $10=$ dolor extremo $)$. El paciente marca la línea que representa el nivel de intensidad del dolor que tiene el día de la entrevista.

\section{Entrevista neuropsiquiátrica internacional (MINI)}

La entrevista neuropsiquiátrica internacional (MINI) es una entrevista diagnóstica heteroaplicada con un coeficiente kappa de 0,69, una sensibilidad del $89 \%$ y un especificidad del $92 \%$ (25). Es una entrevista estructurada de duración breve que explora, para detección y/u orientación diagnóstica, los principales trastornos psiquiátricos del eje-I del DSM-IV y CIE-10. Se encuentra dividida en módulos, identificados por letras, cada uno correspondiente a una categoría diagnóstica. En nuestro caso utilizamos los apartados de trastorno depresivo mayor y trastorno de ansiedad generalizada para determinar si había o no diagnóstico de dichos trastornos. Se utilizó la versión en español (26).

\section{Cuestionario de Pittsburg de Calidad de Sueño (PSQI)}

Se trata de un cuestionario autoadministrado que evalúa la calidad del sueño. Presenta una consistencia interna de 0,81 (coeficiente alfa de Cronbach), coeficiente kappa de 0,61 , sensibilidad de $88,6 \%$, especificidad de $75 \%$ y valor predictivo de la prueba de 80,7\% (27). Consta de un total de 24 ítems, aunque el índice de calidad de sueño se calcula basándose sólo en 19 de ellos. Los 19 ítems se agrupan en siete dimensiones: 1- Calidad del sueño subjetiva, 2- Latencia del sueño, 3- Duración del sueño, 4- Eficiencia habitual del sueño, 5- Perturbaciones del sueño, 6- Uso de medicación para el sueño, 7- Disfunción diurna. La puntuación global se calcula sumando las puntuaciones en las 7 dimensiones y el rango posible va de 0 a 21 , siendo el punto de corte 5/6 puntos. Puntuaciones menores de 5 indican una buena calidad del sueño, mientras que puntuaciones mayores a 6 sugieren una mala calidad. El marco de referencia temporal es el mes previo. Se utilizó la versión española del cuestionario (28).

\section{Escala de Depresión de Montgomery-Asberg (MADRS)}

Se trata de una escala heteroaplicada, con una consistencia interna que oscila entre los valores 0,76 y 0,95 y un coeficiente kappa de 0,80-0,95 (29). La prueba consta de 10 ítems que evalúan el perfil sintomatológico y la gravedad del cuadro depresivo. La puntuación de cada ítem oscila entre 0 y 6 . Proporciona una puntuación global que se obtiene sumando la puntuación asignada en cada uno de los 10 ítems. La puntuación total es un número comprendido entre 0 y 60 puntos, en los que puntuaciones entre 0 y 6 indica ausencia de depresión; 7-19, depresión menor; 20-34, depresión moderada; 35-60, depresión grave. Se utilizó la versión validada en español (30).

\section{Análisis estadísticos}

Se realizó un análisis descriptivo (media y desviación estándar) para las variables continuas y un análisis de frecuencias y porcentajes para las variables ordinales y nominales. Dado que la muestra no cumplía criterios de normalidad, se utilizó la prueba no paramétrica para llevar a cabo los análisis: la prueba U-Mann Whitney para comparar las variables cuantitativas mientras que el análisis de las variables cualitativas se realizó mediante la prueba $\chi^{2}$.

El análisis de correlación entre las variables se realizó a través de la correlación de Pearson. El análisis de regresión lineal múltiple, usando el método por defecto Introducir, se empleó para evaluar la fuerza relativa de los efectos de la edad, género, tipo de tratamiento, valoración del dolor, la puntuación total del cuestionario MADRS y el trastorno de ansiedad generalizada en la puntuación global del PSQI. Para todos los análisis estadísticos, una $\mathrm{p}<0,05$ se consideró estadísticamente significativa. Los análisis se realizaron utilizando el software estadístico programa SPSS 21.0 para Windows. 


\section{RESULTADOS}

\section{Descripción de la muestra}

Un total de 39 pacientes participaron en el estudio. Las características sociodemográficas y clínicas pueden verse en la tabla I. La muestra fue predominantemente femenina $(74,4 \%)$ y la edad media se sitúa en 63,08 años (DE = $12,34)$. El 10,3 \% de la muestra consumía alcohol diariamente y el 48,7 \% cafeína. La media de actividad física es de 2,72 puntos (en un rango del 1 al 6), la media de la valoración del dolor es de 6,11 sobre 10. Un 69,2\% de la muestra presentaba un trastorno depresivo mayor y/o un $61,5 \%$ un trastorno de ansiedad generalizada. La evaluación de la calidad del sueño, recogida por el PSQI, muestra una media de $13(\mathrm{DE}=5)$.

\section{Calidad del sueño, evaluación del dolor y depresión}

Los diferentes ítems del PSQI pueden verse en la tabla II. Existen diferencias estadísticamente significativas en calidad subjetiva del sueño (media $=2,22, \mathrm{DE}=0,801 \mathrm{vs}$. media $=$ $1,17, \mathrm{DE}=0,937 ; \mathrm{p}=0,003)$, en las perturbaciones del sueño $($ media $=2,19, \mathrm{DE}=0,622 v s$. media $=1,5, \mathrm{DE}=0,674$; $\mathrm{p}=0,015$ ), en la utilización de fármacos para dormir (media $=2,52, \mathrm{DE}=1,051$ vs. media $=1, \mathrm{DE}=1.348 ; \mathrm{p}=0,003) \mathrm{y}$ en el de disfunción durante el día (media $=1,84, \mathrm{DE}=1,21$ $v s$. media $=0,42, \mathrm{DE}=0,793 ; \mathrm{p}=0,002$ ). Los pacientes con trastorno depresivo mayor muestran medias más elevadas en estos ítems respecto a aquellos pacientes que no presentan dicho trastorno. También hay diferencias estadísticamente significativas en la puntuación total del PSQI: los pacientes con depresión presentan una puntuación mayor que los pacientes sin este trastorno (media $=15,04, \mathrm{DE}=4,128 \mathrm{vs}$. media $=8,42, \mathrm{DE}=3,630 ; \mathrm{p}=0,000)$.
TABLA I. DATOS SOCIODEMOGRÁFICOS, DE ESTILO DE VIDA Y VARIABLES CLÍNICAS

\begin{tabular}{lcc}
\hline & \multicolumn{2}{c}{ Total $n=39(100 \%)$} \\
\hline \multicolumn{1}{c}{ Variables } & $n$ & Media $(\mathrm{DE})$ o \% \\
\hline Edad & 39 & $63,08(12,34)$ \\
\hline Sexo & & \\
Hombre & 10 & $25,6 \%$ \\
Mujer & 29 & $74,4 \%$ \\
\hline Consumo de alcohol & & \\
No & 35 & $89,7 \%$ \\
Sí & 4 & $10,3 \%$ \\
\hline Consumo de cafeína & & \\
No & 20 & $51,3 \%$ \\
Sí & 19 & $48,7 \%$
\end{tabular}

\begin{tabular}{lcc}
\hline Consumo de tabaco & & \\
Fumador & 5 & $12,8 \%$ \\
No fumador & 21 & $53,8 \%$ \\
Ex fumador & 13 & $33,3 \%$ \\
\hline Actividad física & 39 & $2,72(1,58)$ \\
\hline Valoración del dolor & 39 & $6,11(2,32)$ \\
\hline Diagnóstico psiquiátrico & & \\
TDM $^{1}$ & 27 & $69,2 \%$ \\
TDM $^{1}$ recurrente & 9 & $23,1 \%$ \\
TAG $^{2}$ & 24 & $61,5 \%$ \\
\hline Tratamiento farmacológico & & \\
Antidepresivos & 18 & $46,2 \%$ \\
Hipnóticos & 23 & $59 \%$ \\
Opioides mayores & 24 & $61,53 \%$ \\
Opioides menores & 9 & $23,07 \%$ \\
\hline MADRS $^{3}$ & 39 & $24,62(13,72)$ \\
\hline PSQI $^{4}$ & 39 & $13(5)$ \\
\hline
\end{tabular}

${ }^{1}$ Trastorno depresivo mayor. ${ }^{2}$ Trastorno de ansiedad generalizada. ${ }^{3}$ Escala de depresión de Montgomery-Asberg. ${ }^{4}$ Cuestionario de Pittsburgh de Calidad del Sueño.

TABLA II. CALIDAD DEL SUEÑO EN PACIENTES CON Y SIN TRASTORNO DEPRESIVO MAYOR

\begin{tabular}{lccccc}
\hline & $\begin{array}{c}\text { Total } \\
n=39\end{array}$ & $\begin{array}{c}\text { Trastorno depresivo } \\
\text { mayor } n=27\end{array}$ & $\begin{array}{c}\text { Ausencia de trastorno } \\
\text { depresivo mayor } n=12\end{array}$ & $\begin{array}{c}\text { Significación } \\
\text { estadística }\end{array}$ \\
\hline Media $(D E)$ & Media $(D E)$ & Media $(D E)$ & Test & Valor $p$ \\
\hline Calidad subjetiva del sueño & $1,90(0,968)$ & $2,22(0,801)$ & $1,17(0,937)$ & $\mathrm{U}=256,5$ & $0,003^{*}$ \\
Latencia del sueño & $1,56(1,373)$ & $1,78(1,396)$ & $1,08(1,240)$ & $\mathrm{U}=209,5$ & 0,150 \\
Duración del sueño & $2,03(1,112)$ & $2,19(1,111)$ & $1,67(1,073)$ & $\mathrm{U}=210,5$ & 0,142 \\
Eficiencia habitual de sueño & $2,15(1,226)$ & $2,30(1,203)$ & $1,83(1,267)$ & $\mathrm{U}=202,5$ & 0,221 \\
Perturbaciones del sueño & $1,97(0,707)$ & $2,9(0,622)$ & $1,50(0,674)$ & $\mathrm{U}=241,00$ & $0,015^{*}$ \\
Utilización de fármacos para dormir & $2,05(1,337)$ & $2,52(1,051)$ & $1(1,348)$ & $\mathrm{U}=256,5$ & $0,003^{*}$ \\
Disfunción durante el día & $1,38(1,269)$ & $1,81(1,210)$ & $0,42(0,793)$ & $\mathrm{U}=262,5$ & $0,002^{*}$ \\
Puntuación total & $13(5,005)$ & $15.04(4,128)$ & $8,42(3,630)$ & $\mathrm{U}=289$ & $0,000^{*}$ \\
\hline
\end{tabular}


En la tabla III se observa la evaluación del dolor en diferentes grupos (trastorno depresivo mayor $v s$. ausencia de trastorno depresivo mayor; trastorno de ansiedad generalizada $v s$. ausencia de trastorno de ansiedad generalizada; trastorno depresivo mayor $v s$. trastorno depresivo mayor + trastorno de ansiedad generalizada). Se encontraron diferencias estadísticamente significativas respecto a la evaluación del dolor en el grupo de pacientes con trastorno depresivo mayor $v s$. pacientes sin trastorno depresivo mayor. Los pacientes con depresión presentaban una media superior al grupo de los pacientes que no presentaban dicho trastorno ( media $=6,8, \mathrm{DE}=1,94$ vs. media $=4,37, \mathrm{DE}=2,22$; p: 0,002). No se encontraron diferencias estadísticamente significativas en el resto de grupos.

\section{Calidad del sueño según tratamiento farmacológico}

El $61,5 \%$ de la muestra estaba siendo tratado con opioides mayores mientras que el 38,5\% restante lo estaba siendo con opioides débiles y/u otros fármacos para tratar el dolor. No existen diferencias estadísticamente significativas en la calidad del sueño entre ambos grupos (Tabla IV).

\section{Resultados de los análisis de la correlación}

La correlación de Pearson se utilizó para examinar las relaciones bivariadas entre las variables edad, evaluación del dolor, puntuación global del cuestionario PSQI y puntuación global del cuestionario MADRS.

Una buena calidad del sueño correlacionó significativamente con la edad $(\rho=-0,449, p=0,01)$, mientras que una mala calidad del sueño correlacionó significativamente con la gravedad de la sintomatología depresiva ( $\rho=0,600$, $\mathrm{p}=0,01)$ pero no con la evaluación del dolor $(\rho=0,234$, $\mathrm{p}=0,01)($ Tabla V).

\section{Resultados del análisis de la regresión lineal múltiple}

Finalmente, se realizó un análisis de regresión lineal múltiple con el método "Introducir" para analizar el efecto de las variables independientes estudiadas en la calidad del

TABLA III. EVALUACIÓN DEL DOLOR EN DIFERENTES GRUPOS DE PACIENTES

\begin{tabular}{|c|c|c|c|}
\hline & Media $(D E)$ & U de Mann-Whitney & Valor $p$ \\
\hline Trastorno depresivo mayor $(\mathrm{n}=27)$ & $6,89(1,94)$ & 259,500 & $0,002 *$ \\
\hline Ausencia de trastorno depresivo mayor $(\mathrm{n}=12)$ & $4,37(2,22)$ & & \\
\hline Trastorno de ansiedad generalizada $(n=24)$ & $6,06(2,63)$ & 182,500 & 0,943 \\
\hline Ausencia de trastorno de ansiedad generalizada $(\mathrm{n}=15)$ & $6,20(1,82)$ & & \\
\hline Trastorno depresivo mayor $(\mathrm{n}=9)$ & $6,67(1,93)$ & 91,000 & 0,631 \\
\hline Trastorno depresivo mayor + trastorno de ansiedad generalizada $(n=18)$ & $7(2)$ & & \\
\hline
\end{tabular}

$* \mathrm{p}<0,05$

TABLA IV. CALIDAD DEL SUEÑO Y USO DE OPIOIDES

\begin{tabular}{ccccc}
\hline & Opioides mayores $n=24$ & Opioides débiles y/u otros $n=15$ & \multicolumn{2}{c}{ Significación estadística } \\
\hline Ítems del PSQI & \% Media $(\mathrm{DE})$ o $\%$ & \% Media $(\mathrm{DE}) o \%$ & Test & Valor $p$ \\
\hline Calidad subjetiva & $1,83(1,09)$ & $2(0,756)$ & $\mathrm{U}=191,500$ & 0,743 \\
Latencia & $1,54(1,285)$ & $1,60(1,549)$ & $\mathrm{U}=179,500$ & 0,989 \\
Duración & $1,83(1,167)$ & $2,33(0,976)$ & $\mathrm{U}=225,500$ & 0,191 \\
Eficiencia & $2,13(1,262)$ & $2,20(1,207)$ & $\mathrm{U}=180,000$ & 1 \\
Perturbaciones & $1,96(0,751)$ & $2(0,655)$ & $\mathrm{U}=181,500$ & 0,966 \\
Medicación & $2,33(1,204)$ & $1,6(1,454)$ & $\mathrm{U}=128,000$ & 0,138 \\
Disfunción diurna & $1,21(1,141)$ & $1,67(1,447)$ & $\mathrm{U}=216,500$ & 0,296 \\
Puntuación total & $12,75(5,160)$ & $13,40(4,896)$ & $\mathrm{U}=194,000$ & 0,700 \\
\hline
\end{tabular}

${ }^{1}$ Cuestionario de Pittsburgh de Calidad del Sueño. 
TABLA V. CORRELACIONES ENTRE DISTINTAS VARIABLES ESTUDIADAS

\begin{tabular}{llll}
\hline & Edad & EVA & MADRS \\
\hline EVA $^{1}$ & $-0,201$ & & \\
MADRS $^{2}$ & $-0,147$ & 0,288 & \\
PSQI $^{3}$ & $-0,449 *$ & 0,234 & $0,600^{*}$ \\
\hline
\end{tabular}

*La correlación es significativa al nivel 0,01 (bilateral). ${ }^{1}$ Escala visual analógica. ${ }^{2}$ Escala de depresión de Montgomery-Asberg. ${ }^{3}$ Cuestionario de Pittsburgh de Calidad de Sueño.

sueño, la variable dependiente fue la puntuación global del PSQI (Tabla VI). La gravedad de la depresión y la edad fueron, en este orden, los predictores significantes de la calidad del sueño. El modelo de regresión para el funcionamiento general fue estadísticamente significativo y representó el 40,4\% de la varianza explicada ( $\mathrm{R} 2=0,404, \mathrm{~F}=$ $5,30, \mathrm{p}=0,001)$. No hay evidencias de multicolinealidad en el modelo (FIV <2,5).

\section{DISCUSIÓN}

El objetivo principal de este estudio fue evaluar la calidad del sueño y la prevalencia del trastorno depresivo mayor y trastorno de ansiedad generalizada en una muestra de pacientes con dolor crónico del tipo osteomuscular. Los resultados principales de nuestro estudio están en la línea con los resultados de otros estudios que han investigado la relación de estas variables en los pacientes con dolor crónico (8,31-33).

En primer lugar, más de la mitad de nuestra muestra presentaba un trastorno depresivo mayor y un trastorno de ansiedad generalizada (69,2 y 61,5\%, respectivamente). Estos datos apoyan los resultados de otros estudios, como por ejemplo el de Castro y cols. (34) en el que un 61,5\% de su muestra presentaba depresión y un 72,8 \% ansiedad. La comorbilidad de un trastorno afectivo en pacientes con dolor crónico es bastante alta, aunque el porcentaje es amplio, se sitúa entre el 22 y el 78 \% (22). Además de presentar altos niveles de depresión, el sueño también se ve influido de manera negativa. La calidad del sueño es peor en aquellos pacientes con dolor crónico respecto a sujetos controles (31). En nuestro caso, no comparamos con sujetos sanos, aunque la media de nuestros pacientes se situaba en 13, indicando también una pobre calidad del sueño. Son muchos los estudios que han intentado determinar las causas de esta relación. Algunos de ellos asocian la mala calidad del sueño a la intensidad del dolor (35), a los trastornos del estado de ánimo, concretamente a la depresión (31) e investigaciones más recientes lo asocian a la administración crónica de opioides potentes $(8,36,37)$. En nuestro estudio, evaluamos la influencia de los opioides potentes en la calidad del sueño. Según nuestro conocimiento, este estudio es el primero en diferenciar a los pacientes según el tipo de opioide tomado (potente o débil). No encontramos diferencias estadísticamente significativas en la calidad del sueño entre ambos grupos. Una posible explicación podría ser que nuestra muestra no fuera lo suficientemente amplia como para observar los efectos. Por otra parte, sí encontramos diferencias significativas cuando evaluábamos la calidad del sueño en pacientes con depresión y los que no presentaban este diagnóstico. Estos resultados van en la misma dirección que otros estudios en los que demuestran que la depresión va ligada a una mala calidad del sueño en pacientes con dolor crónico (31).

En nuestro estudio observamos una correlación positiva estadísticamente significativa entre la gravedad de la sintomatología depresiva y la evaluación del dolor. Estos datos sugerirían una posible relación bidireccional entre ambas variables $(22,38)$.

Finalmente, cuando se realizaron los análisis de la regresión lineal múltiple algunos de nuestros resultados fueron reforzados. En conjunto, el modelo representa el 40,4 \% de la varianza en la calidad del sueño atribuido a la gravedad de la depresión y la edad. Por tanto, en nuestro caso, son la

TABLA VI. ANÁLISIS DE LA REGRESIÓN LINEAL MÚLTIPLE VARIABLE DEPENDIENTE: PUNTUACIÓN GLOBAL PSQI

\begin{tabular}{lccccccc}
\hline \multicolumn{7}{c}{ IC de 95,0 \% para $\beta$} \\
\hline & $\beta$ & $p$ & $\begin{array}{c}\text { Limite } \\
\text { inferior }\end{array}$ & $\begin{array}{c}\text { Limite } \\
\text { superior }\end{array}$ & $R^{2}$ corregida & $\begin{array}{c}\text { Cambio } \\
\text { en } R^{2}\end{array}$ & Sig. \\
\hline Trastorno de ansiedad generalizada & $-0,064$ & 0,664 & $-3,667$ & 2,369 & 0,040 & 0,066 & 0,116 \\
Puntuación global MADRS & 0,572 & $0,000^{*}$ & 0,102 & 0,315 & 0,342 & 0,360 & $0,000^{*}$ \\
Valoración del dolor & $-0,020$ & 0,886 & 0,651 & 0,564 & 0,029 & 0,055 & 0,152 \\
Tipo de tratamiento & 0,040 & 0,767 & $-2,395$ & 3,216 & $-0,023$ & 0,004 & 0,699 \\
Género & 0,048 & 0,723 & $-2,530$ & 3,608 & 0,015 & 0,011 & 0,517 \\
Edad & $-0,410$ & $0,009^{*}$ & $-0,288$ & $-0,044$ & 0,180 & 0,202 & $0,004^{*}$ \\
\hline
\end{tabular}

${ }^{1}$ Escala de depresión de Montgomery. ${ }^{*} \mathrm{p}<0,05$. 
edad y la gravedad del trastorno depresivo los predictores significantes de la mala calidad del sueño. Estos resultados coinciden parcialmente con Sayar y cols. (39), en el que en su modelo, la depresión, entre otras variables, también es un predictor de la calidad del sueño.

Este estudio presenta una serie de limitaciones. El tamaño muestral es pequeño comparado con otros estudios previos, ello hace que el poder estadístico sea menor. Otra limitación es que sólo hemos incluido dolor crónico tipo osteomuscular, pudiendo limitar el universo muestral. Además, el estudio sólo incluye autoinformes del paciente, sin ninguna otra información de familia y/o amigos. A pesar de estas limitaciones, el trabajo presenta importantes fortalezas: es el primer estudio en contrastar grupos en función de los opioides que toman (fuertes vs. débiles) y se evalúa no sólo depresión, sino también ansiedad y la comorbilidad de ambos.

Futuras investigaciones destinadas a la evaluación de la calidad del sueño y la prevalencia de trastornos afectivos en pacientes con dolor crónico serán cruciales para entender los factores que envuelven a esta población y así poder mejorar el manejo clínico, su calidad de vida e impacto social.

\section{CORRESPONDENCIA:}

Adoración Castro

Institut Universitari d'Investigació en Ciències de la Salut

(IUNICS)

Universitat de les Illes Balears

Ctra. Valldemossa, km 7,5

07122 Palma de Mallorca

e-mail: a.castro@uib.es

\section{BIBLIOGRAFÍA}

1. Sessle, Barry J. What is pain, and why and how do we experience pain? En: Lavigne E, Sessle BJ, Choinière M, Soja PJ, editors. Sleep and Pain. Seattle: IASP Press; 2007. p. 23-43.

2. Casals M, Samper D. Epidemiología, prevalencia y calidad de vida del dolor crónico no oncológico. Estudio ITACA. Rev Soc Esp Dolor 2004;11:260-9.

3. Català E, Reig E, Artes M, Aliaga L, López JS, Segú JL. Prevalence of pain in the Spanish population: Telephone survey in 5000 homes. Eur J Pain 2002;6:133-40.

4. IASP: Subcommittee on Taxonomy. Classification of chronic pain. Descriptions of chronic pain syndromes and definitions of pain terms Pain 1986;Supl. 3:S1-S226.

5. Soucase B, Monsalve V, Soriano JF. Afrontamiento del dolor crónico: el papel de las variables de valoración y estrategias de afrontamiento en la predicción de la ansiedad y la depresión en una muestra de pacientes con dolor crónico. Rev Soc Esp Dolor 2005;12:8-16.

6. Llorca GJ, Muriel C, González Tablas MM, Díez MA. Relación entre características del dolor crónico y niveles de depresión. Rev Soc Esp Dolor 2007;1:26-35.
7. Souza FAEF, Silva JA. Avaliação e mensuração de dor em contextos clínicos e de pesquisa. Rev Dor 2004;5:408-29.

8. Mencías Hurtado AB, Rodríguez Hernández JL. Trastornos del sueño en el paciente con dolor crónico. Rev Soc Esp Dolor 2012;19(6):332-4.

9. Moldofsky H. Sleep and pain. Sleep Med Rev 2001;5:38596.

10. Wilson KG, Eriksson MY, D’Eon JL, Mikail SF, Emery PC. Major depression and insomnia in chronic pain. Clin J Pain 2002;18:77-83.

11. Okifuji A, Hare BD. Do sleep disorders contribute to pain sensivity? Curr Rheumatol Rep 2011;13(6):528-34.

12. Lavigne GJ, Nashed A, Manzini C, Carra MC. Does sleep differ among patients with common musculoskeletal pain disorders? Curr Rheumatol Rep 2011;13(6):535-42.

13. Calandre EP, Rico-Villademoros F, Rodríguez-López CM. Monotherapy or combination therapy for fibromyalgia treatment? Curr Rheumatol Rep 2012;14(6):568-75.

14. Toms J. Updated view of fibromyalgia. Cas Lek Cesk 2012;151(9):415-9.

15. Morin CM, Gibson D, Wade J. Self-Reported sleep and mood disturbance in chronic pain patients. Clin J Pain 1998;14:311-4.

16. Smith MT, Perlis ML, Smith MS, Giles DE, Carmody TP. Sleep quality and presleep arousal in chronic pain. J Behav Med 2000;23:1-13.

17. Shaw PJ, Cirelli C, Greenspan RJ, Tononi G. Correlates of sleep and waking in drosophila melanogaster. Science 2000;271:216-8.

18. Rosenberg J. Sleep disturbances after non-cardiac surgery. Sleep Med Rev 2001;5:129-37.

19. Velázquez I, Muñoz M, Sánchez M, Zenner A, Velázquez L. Trastornos del sueño e incidencia de ansiedad y depresión en pacientes con dolor crónico no maligno tratados con opioides potentes. Rev Soc Esp Dolor 2012;19(2):49-58.

20. Fishman SM, Ballantyne JC, Rathmell JP. Bonica's Management of Pain. $4^{\text {th }}$ ed. Philadelphia: Wolters-Kluwer/Lippincott Williams \& Wilkins; 2010.

21. Bras M, Dordević V, Gregurek R, Bulajić M. Neurobiological and clinical relationship between psychiatric disorders and chronic pain. Psychiatr Danub 2010;22(2):221-6.

22. Morales-Vigil T, Alfaro-Ramírez O, Sánchez S, Vázquez-Pineda F. Ansiedad y depresión por dolor neuropático y nocioceptivo. Rev Med Inst Mex Seguro Soc 2008;46(5):479-84.

23. Plata-Muñoz ME, Castillo-Olivares ME, Guevara-López U. Evaluación de afrontamiento, depresión, ansiedad e incapacidad funcional en pacientes con dolor crónico. Rev Mexicana Anestesiología 2004;27(1).

24. Tennen H, Affleck G, Zautra A. Depression history and coping with chronic pain: A daily process analysis. Health Psychol 2006;25(3):370-9.

25. Lecrubier Y, Sheehan DV, Weiller E, Amorim P, Bonora I, Harnett K, et al. The Mini International Neuropsychiatric Interview (MINI). A short diagnostic structured interview: Reliability and validity according to the CIDI. Eur Psychiatry 1997;12(5):224-31.

26. Ferrando L, Bobes J, Gibert J, Soto M, Soto O. Mini International Neuropsychiatric Interview. Versión en español 5.0.0. 2000: Madrid.

27. Buysse DJ, Reynolds CF, Monk TH, Berman SR, Kupfer DJ. The Pittsburgh Sleep Quality Index: A new instrument for psychiatric research and practice. Psychiatry Res 1989;28(2):193-213. 
28. Macías Fernández JA, Royuela Rico A. La versión española del índice de calidad de sueño de Pittsburgh. Informaciones Psiquiátricas 1996;146:465-72.

29. Montgomery SA, Asberg M. A new depression scale designed to be sensitive to change. Br J Psychiatry 1979;134(4):382-9.

30. Lobo A, Chamorro L, Luque A, Dal-Ré R, Badia X, Baró E. Validación de las versiones en español de la montgomery Anxiety Rating Scale para la evaluación de la depresión y de la ansiedad. Med Clínica 2002;118(13):493-9.

31. Naughton F, Ashworth P, Skevington SM. Does sleep quality predict pain-related disability in chronic pain patients? The mediating roles of depression and pain severity. Pain 2007;127(3):243-52.

32. Calsina-Berna A, Moreno N, González-Barboteo J, Solsona L, Porta J. Frequency of pain as a reason for visiting a primary care clinic and its influence on sleep. Aten Primaria 2011;43(11):568-75.

33. Wong WS, Fielding R. The eco-morbidity of chronic pain, insomnia, and fatigue in the general adult population of
Hong Kong: Prevalence and associated factors. J Psychosom Res 2012;73:28-34.

34. Castro M, Daltro C. Sleep patterns and symptoms of anxiety and depression in patients with chronic pain. Arq Neuropsiquiatr 2009;67(1):25-8.

35. Gálvez R, Marsal C, Vidal J, Ruiz M, Rejas J. El dolor neuropático como causa de ansiedad, depresión y trastornos del sueño en condiciones de práctica lemédica habitual: resultados del estudio DONEGA. Rev Soc Esp Dolor 2006;13(2):81-95.

36. Onen SH, Onen F, Courpron P, Dubray C. How pain and analgesics disturb sleep. Clin J Pain 2005;5:385-96.

37. Miaskowski C. Pharmacologic management of Sleep Disturbances in Noncancer-Related Pain. Pain Manag Nurs 2009;10:3-13.

38. Means-Christensen AJ, Roy-Byrne PP, Sherbourne CD, Craske MG, Stein MB. Relationships among pain, anxiety and depression in primary care. Depress Anxiety. 2008;25(7):593-600.

39. Sayar K, Arikan M, Yontem T. Sleep quality in chronic pain patients. Can J Psychiatry 2002;47(9):844-8. 